19 Revue d'histoire du XIXe siècle

Société d'histoire de la révolution de 1848 et des

révolutions du XIXe siècle

$24 \mid 2002$

Varia

\title{
Le romancier, lecteur du social dans la France de la Monarchie de Juillet
}

Judith Lyon-Caen

\section{OpenEdition}

Journals

Édition électronique

URL : http://journals.openedition.org/rh19/367

DOI : $10.4000 /$ rh 19.367

ISSN : 1777-5329

Éditeur

La Société de 1848

Édition imprimée

Date de publication : 1 juin 2002

Pagination : 15-32

ISSN : 1265-1354

Référence électronique

Judith Lyon-Caen, «Le romancier, lecteur du social dans la France de la Monarchie de Juillet», Revue d'histoire du XIXe siècle [En ligne], 24 | 2002, mis en ligne le 05 mars 2008, consulté le 10 décembre 2020. URL : http://journals.openedition.org/rh19/367 ; DOI : https://doi.org/10.4000/rh19.367

Ce document a été généré automatiquement le 10 décembre 2020.

Tous droits réservés 


\title{
Le romancier, lecteur du social dans la France de la Monarchie de Juillet
}

\author{
Judith Lyon-Caen
}

\section{NOTE DE L'ÉDITEUR}

Une première version de cet article a été présentée à l'Université Paris 7 dans le cadre du séminaire de Marie-Noëlle Bourguet et de Dominique Kalifa, «Explorations, enquêtes, descriptions ». L'auteur tient à les remercier, ainsi que tous les participants du séminaire, pour leurs critiques et leurs suggestions.

1 Dans Soixante ans de souvenirs, Ernest Legouvé, le demi-frère d'Eugène Sue, rapporte que le romancier, après avoir rédigé et publié quelques chapitres des Mystères de Paris, au début de l'été 1842, aurait été comme révélé à lui-même par un article du fouriériste Victor Considérant, qui affirmait : «Je vois où va l'auteur ! Il entre dans une voie inexplorée ! Il entreprend la peinture des souffrances et des besoins des classes travailleuses ». Le romancier aurait alors rencontré le directeur de la Phalange et aurait déclaré, au terme de l'entrevue : «je vois clair ». Et Legouvé poursuit : «[Eugène Sue] se lança dans le monde d'en bas comme il s'était lancé dans le monde d'en-haut. À la place de l'habit rouge du chasseur, à la place du bouton des grandes vèneries, des camélias à la boutonnière, il acheta une casquette, une blouse, de gros souliers, et il s'en alla, le soir, dans les faubourgs, dans les cabarets de barrière, dans les réunions d'ouvriers, dans les garnis, dans les taudis, dans les hospices, et plongeant pour ainsi dire son imagination au milieu de toutes ces misères, de toutes ces haines, de tous ces dévouements " ${ }^{1}$.

2 L'anecdote a souvent été reprise par les biographes de Sue, pour lesquels les souvenirs de Legouvé constituent une source essentielle. Nora Atkinson, dans son ouvrage de 1929, Eugène Sue et le roman-feuilleton, en donne une variante plus concise, mais significative : "Sue lit l'article [de Considérant] et va causer avec l'auteur. Il voit clair. Alors, il prend une casquette, une blouse, de gros souliers, et il s'en va, le soir, à pied dans la Cité, dans 
les cabarets et les tapis-francs, dans les garnis, étudiant partout la vie de ces malheureux qu'il va décrire ${ }^{2}$.

3 En suivant l'anecdote de Legouvé - qui reste parfaitement invérifiable -, Nora Atkinson campe le romancier en déchiffreur de cette zone ombreuse et fascinante de la société de la monarchie de Juillet que constituent les quartiers populaires du centre de Paris. Dans la logique de la biographie, cet épisode sert, d'une part, à rendre compte du tournant "social» d'Eugène Sue, qui se défait de l'habit du dandy sceptique pour devenir défenseur des classes laborieuses. L'anecdote permet, d'autre part, de rapprocher le romancier du héros des Mystères de Paris, le prince Rodolphe de Gérolstein, qui quitte sa petite principauté allemande pour plonger, déguisé en ouvrier, dans un Paris misérable et dangereux. Dans la version de Nora Atkinson, le monde dans lequel pénètre le romancier ressemble d'abord au décor parisien des premiers chapitres des Mystères de Paris: le quartier de la Cité et ses « tapis-francs » - ces cabarets louches dont le nom d'argot est popularisé, précisément, par le succès des Mystères de Paris.

L'anecdote de Legouvé déploie ainsi une scénographie du romancier " lecteur du social », qui s'enroule curieusement autour de l'œuvre en train de s'écrire : au moment supposé de cet épisode, Eugène Sue avait alors tout juste rédigé et publié quelques feuilletons des Mystères de Paris dans le Journal des Débats; le roman restait un chantier ouvert, un vaste ensemble de possibles. La pratique de l'observation sociale chez Eugène Sue ne procéderait pas du projet d'écriture initial mais de la réception d'une première partie de son texte par un «socialiste " - c'est-à-dire, pour revenir à un usage littéral du terme, par l'un de ceux qui mettent la "question sociale»-, celle de la misère des classes laborieuses, au cœur de leur pensée sur le monde contemporain. Reprenant à son compte un programme énoncé lors de la réception des premiers feuilletons de son roman, déroulant un projet formulé hors du lieu auctorial, Sue aurait été comme entraîné par la dynamique de son œuvre.

5 La figure de romancier que construit cette anecdote est inhabituelle : s'il est courant qu'un biographe d'écrivain cherche à nourrir celui-ci de la chair de ses héros - faute de sources plus solides sur l'homme -, il est moins fréquent de substituer à l'inspiration créatrice du romancier une exploration in situ, qui rapproche l'écrivain de cette figure devenue familière à l'époque même de la publication des Mystères de Paris: celle de l'observateur social, qui descend également dans les bas-fonds et les quartiers pauvres des grandes villes. À pousser l'interprétation de l'anecdote, l'identité générique de cet emblème du roman populaire que sont les Mystères de Paris, se brouille : ne s'agit-il pas, au fond, d'une grande enquête sociale mise en roman? Ne faut-il pas sortir les Mystères de Paris et, plus largement tous ces romans de la monarchie de Juillet qui s'attachent à représenter la société contemporaine, du champ clos de la «littérature» pour les rapprocher du plus large ensemble de pratiques qui s'appliquent alors à explorer et à "mettre en texte» le monde contemporain, comme les célèbres enquêtes sociales de Villermé, de Buret ou de Frégier ${ }^{3}$ ?

6 On peut partir de cette mise en scène d'Eugène Sue en explorateur de la société de son temps pour appréhender les romans de la monarchie de Juillet, tels qu'ils sont donnés par leurs auteurs et reçus par leurs lecteurs, comme une écriture du monde social, au même titre que d'autres formes d'écriture et de savoir, comme les enquêtes sociales ou tous ces tableaux de Paris et «physiologies » de types sociaux qui prolifèrent dans les années 1840. $\mathrm{Au}$ cours des années 1830, au moment même où l'espace de la littérature est envahi par le genre romanesque, la société contemporaine semble devenir le champ d'investigation 
privilégié du roman, qui entre massivement en "régime réaliste». Il ne s'agit pas ici d'évaluer cette pertinence ni de reprendre le vieux débat sur l'authenticité des descriptions de la société parisienne, dans la Comédie humaine par exemple. La littérature romanesque constitue ici l'objet et non le moyen du questionnement historique : loin de tout usage documentaire de ces textes romanesques, à la charnière de l'histoire littéraire et de l'histoire, nous voudrions tenter ici de réfléchir aux enjeux de la représentation du monde social dans un grand nombre de romans de la Monarchie de Juillet, à partir d'une analyse de la "pratique romanesque » et de la réception de ces deux romanciers majeurs que sont Balzac et Eugène Sue. Comme le suggère indirectement l'anecdote de Legouvé sur Sue, de nombreux romans des années 1830-1848 proposent, selon certains procédés, de «mettre en texte » le monde social pour le donner à lire : nous montrerons qu'ils se situent, d'une part, au cœur d'une entreprise collective d'écriture du social, partageant avec d'autres types de textes une interrogation sur la manière de représenter la complexité du monde contemporain ainsi que des modes d'écriture spécifiques. Ces romans semblent, d'autre part, être reçus par leurs lecteurs, critiques de profession ou grand public anonyme, comme des représentations pertinentes du monde social contemporain: dans les appropriations qu'en réalisent leurs lecteurs, la vérité romanesque se situe du côté de la réalité sociale et les romans permettent de rendre intelligibles les mécanismes sociaux complexes souvent perçus comme opaques.

7 On sait que le roman acquiert, sous la monarchie de Juillet, une place centrale au sein de la production imprimée. Les ouvrages religieux et moraux, les livres d'éducation et le fonds « classique » (La Fontaine, Molière, Racine...). continuent de représenter les tirages les plus élevés, mais les romans gagnent en visibilité, à la fois par le nombre d'ouvrages nouveaux et par la diffusion que leur assure les cabinets de lecture, en dépit de tirages moyens assez faibles. Edmond Werdet, qui fut notamment l'éditeur de Balzac, date précisément de 1830 cette irruption du roman, et témoigne, a posteriori, du sentiment d'invasion de la librairie par le genre romanesque : «Ce nouveau genre de littérature eut une telle expansion dès la fin de 1830, que la France entière sembla ne plus former qu'un immense salon de lecture, où chacun attendait avec impatience son tour pour dévorer l'œuvre fraîchement éclose. [...] La librairie de romans et de nouveautés prit tout à coup un développement immense. [...] À côté de cette rage de romans qui envahissait la France entière, les vieux classiques de la bonne littérature, ces fidèles amis de la jeunesse de tout homme qui a reçu une éducation solide, ces chefs-d'œuvre qui feront éternellement la gloire de notre nation, restaient relégués et presque oubliés au fond des magasins » ${ }^{4}$.

8 Au cours des années 1840 , le succès des romans-feuilletons dans la presse quotidienne et l'invention de formules éditoriales moins coûteuses, comme la vente en livraisons ou le format Charpentier, font accéder certains romans, ceux de Sue et de Dumas surtout, au statut de « best-sellers » : ces succès esquissent le régime de consommation culturelle de masse qui se développe dans la seconde moitié du siècle 5 .

9 Parallèlement à cette expansion quantitative, trois mutations qualitatives sont alors perceptibles : tout en devenant considérable en nombre, le roman comme genre acquiert tout d'abord une certaine respectabilité. En dépit du mépris dont les critiques des grandes revues et des quotidiens accablent les romans nouveaux, malgré la croisade contre la " littérature industrielle ", identifiée surtout au roman-feuilleton, qui se déploie à partir de $1839^{6}$, le genre romanesque, du moins dans ses manifestations passées, acquiert une certaine reconnaissance. Occupant en outre une place croissante dans la production littéraire comme dans les lectures, le roman tient le devant de la scène critique : il suscite 
une intense production polémique, ne serait-ce que parce que la posture de la «critique littéraire ", qui s'invente alors dans la presse, se définit très largement contre l'invasion de l'espace imprimé par le roman. Les lieux qu'investit la critique sont en effet ceux-là mêmes que le roman conquiert: les revues littéraires, comme la Revue de Paris, et les feuilletons de la presse quotidienne, après l'« invention » du roman-feuilleton par Émile de Girardin dans la Presse en 1836. Or le type de relation entre l'œuvre et le public que suppose la diffusion du roman en feuilletons ignore la médiation critique : la qualité d'un roman se juge par la quantité d'abonnés qu'il procure au journal, et donc à la curiosité impatiente qu'il éveille chez le lecteur. Le roman-feuilleton menace ainsi la fonction même du magistère critique: celle de désigner au public les bons romans, selon les critères largement partagés du bon goût et de la morale. La croisade contre le romanfeuilleton et l'industrialisation de la littérature à partir de la fin des années 1830 doit ainsi se comprendre comme une revendication d'existence de la part des critiques. Mais le roman fait scandale bien au-delà des sphères de la critique littéraire : mis en procès lors de l'affaire Lafarge par exemple, les romans nouveaux inspirent également, nous y reviendrons, de longues diatribes à la Chambre au député de Saône-et-Loire, ChapuysMontlaville, qui s'inquiète du désordre social dont ils portent les germes.

10 Cette hantise ne tient pas seulement aux nouveaux modes de diffusion des textes romanesques dans la presse mais aussi aux mutations du champ d'investigation des romans dans les années 1830 et 1840 . Les romans publiés sous la monarchie de Juillet sont en effet majoritairement des textes que leurs contemporains - critiques, éditeurs et romanciers - désignent comme des "romans de mœurs", utilisant un sytème de désignation hérité du XVIII ${ }^{\mathrm{e}}$ siècle. C'est en effet en se plaçant du côté de l'histoire ou des mémoires, en se voulant peinture ou étude des mœurs, «tableau moral et animé de la vie humaine ${ }^{7}$ que le roman a conquis, au XVIII ${ }^{e}$ siècle, une légitimité contre les reproches d'invraisemblance et de frivolité. Mais le domaine recouvert par le terme "mœurs » s'étend considérablement après 1830 . Évoquons seulement les déplacements qu'opère le système des titres balzaciens dans le lexique de l'étude de mœurs. En réunissant ses romans, en 1834, sous le titre Études de mours au XIX siècle, Balzac réalise un renversement: l'indication générique ne vient plus compléter le titre par une information sur le type de roman donné à lire ; devenue sur-titre, elle désigne désormais un projet dont les romans constituent des applications. L'ambition signifiée par cette mutation est confirmée par l'ampleur de la référence temporelle, - « au XIX siècle » -, et par la réunion des romans en sous-ensembles - Scènes de la vie privée, Scènes de la vie de province, Scènes de la vie parisienne -, qui correspondent à un quadrillage du monde. Le même type de déplacement est perceptible lorsque Balzac entreprend de publier chez Delloye en 1838 une édition illustrée de ses œuvres sous le titre Études sociales (seule la Peau de Chagrin est publiée dans ce cadre). En substituant « études sociales » à " études de mœurs", Balzac change de lexique, abandonne les dénominations génériques traditionnelles pour un épithète qui renvoie davantage à la philanthropie ou à l'économie politique : comme, quelques années plus tard, dans la timide diffusion du terme « roman social », les dénominations génériques ne renvoient plus seulement à l'histoire du genre romanesque, mais également à des manières actuelles de réfléchir sur le contemporain.

11 De nombreux romans des années de la Monarchie de Juillet, et certains de ceux qui ont le plus de succès, des Mémoires du Diable aux Mystères de Paris ou au Juif errant, se veulent des romans sur la société contemporaine, des représentations de la société contemporaine. Le plus populaire des romanciers de l'époque, Paul de Kock propose des intrigues qui sont 
autant de guides dans un univers social que le lecteur apprend à déchiffrer; Soulié, dans les Mémoires du Diable en 1839, propose, comme Eugène Sue dans les Mystères de Paris, une exploration des replis du siècle et des " plaies sociales ». Il n'est pas question d'évoquer ici la multitude et la complexité de ces mondes romanesques qui se donnent chacun pour des représentations vraies de la société contemporaine. Il importe en revanche de souligner le caractère collectif de cette revendication, et de montrer comment ces romans s'inscrivent dans une vaste entreprise collective de mise en texte du social.

Il faut en effet replacer ces romans au sein de cette production textuelle aux formes multiples qui entend, sous la monarchie de Juillet, représenter le monde social. Non seulement l'affirmation de l'opacité du monde social issu de la Révolution et de la Restauration est un thème très diffusé, mais cette opacité semble être au principe d'un certain nombre de textes qui visent à déchiffrer, à décrypter, à dévoiler, à représenter le monde social comme un texte lisible, un paysage visible. On peut ainsi associer les romans sur la société contemporaine à la littérature nombreuse des « tableaux de mœurs » et des descriptions de Paris - parmi lesquelles les Physiologies ou la série des Français peints par eux-mêmes, publiée chez Curmer entre 1840 et $1842^{8}-$, comme aux enquêtes sociales suscitées par l'Académie des Sciences morales et politiques à la fin de la décennie 1830 . Malgré la variété des lieux et des milieux dont ils émanent, des registres sur lesquels ils se déploient, des publics et des usages qu'ils visent, tous ces textes ont en commun de constituer la société en objet - opaque, mystérieux, inconnu ou simplement illisible -, pour la déployer, la classer, l'explorer, la dévoiler. En bref, l'inquiétude ou la curiosité que suscitent les brouillages de la société post-révolutionnaire et industrielle nourrissent une sociographie multiforme du monde contemporain.

On peut tout d'abord rapprocher les romans des enquêtes sociales et de cette abondante littérature descriptive que Walter Benjamin avait baptisé, en référence aux spectacles des Panoramas, la "littérature panoramique " ${ }^{9}$, par les projets de description et de connaissance du monde social que formulent ces trois types de textes. Les préfaces balzaciennes développent longuement le thème du brouillage de la société contemporaine et proposent de faire du roman l'instrument de la connaissance du contemporain. Dans l'avant-propos de Riche et pauvre, en 1836, Émile Souvestre propose de la même manière de rendre le roman à «l'empire du réel ", d'en faire «la chambre obscure de la société "; il lance alors un cycle de romans au titre évocateur : les Romans de la vie réelle. L'exploration des "mystères» sociaux ordonne l'économie de nombreux romans-feuilletons, des Mémoires du Diable de Soulié au Juif errant en passant, bien sûr, par les Mystères de Paris. Le roman se donne donc comme un instrument de lecture du social. La littérature panoramique des Physiologies et des séries collectives, comme Paris ou le livre des Cent-et-un (1831), Les Français peints par eux-mêmes (1840-1842) ou le Diable à Paris (1845-1846), part du constat de la radicale complexification de la société française depuis la Révolution pour renouveler le genre du tableau de mœurs et de la description de Paris hérité de Mercier : la complexité nouvelle du monde social exige le recours à une écriture collective, puis à l'illustration, bref à une représentation composite, qui articule le plus grand nombre de manières de dire, de raconter, de peindre le contemporain. Chez Buret ou chez Frégier, l'enquête sociale s'attache à ces points d'opacité du monde social que sont le crime et le paupérisme. La focalisation commune des médecins hygiénistes, des philanthropes, des administrateurs et des économistes sur le crime et la misère rompt avec la statistique sociale des premières décennies du siècle : la société n'apparaît plus seulement comme une masse bigarrée qu'il faut connaître et classer pour la gouverner, 
mais un corps malade, affligé de plaies mal connues et mal comprises, une entité aux fonctionnements complexes. Cette perception et l'urgence d'apprendre à "gouverner la misère" expliquent l'intensification de l'observation sociale et la recherche d'une écriture propre à représenter la misère ${ }^{10}$.

Outre la convergence de ces projets, on peut souligner, en dépit de la variété des lieux de l'écriture et des usages que visent les textes, la proximité des solutions descriptives qu'adoptent romans, enquêtes sociales et textes panoramiques. C'est, en premier lieu, le commun recours à une écriture du type, élément de base de la littérature panoramique ( Physiologies et galeries de types), recours fondamental de l'écriture romanesque, mais également pratique descriptive courante dans l'enquête sociale chez Frégier, et dans une moindre mesure, chez Villermé. Dans la littérature panoramique, la simplification du monde en types est mise au service d'un balayage horizontal de l'espace social ; le même principe d'exhaustivité est à l'œuvre dans la Comédie humaine ou dans les romans feuilletons à mystères, dont l'achèvement semble subordonné à l'épuisement du monde à décrire; il ordonne également l'enquête sociale, même lorsqu'elle se veut circonscrite à une portion de la société : « tout voir, tout connaître, tout entendre ", tel est, on le sait, le programme de Villermé. Mais ce qui, plus encore, rapproche ces textes, c'est une commune appréhension du social comme épaisseur à traverser, univers à décrypter, à dévoiler, appréhension qui donne lieu une « description verticale» ${ }^{11}$. Il faut souligner le constant usage des métaphores organiques et la représentation de la société comme monde ténébreux, corps malade affligé de plaies mystérieuses, que Balzac et Sue partagent avec Parent-Duchâtelet, Frégier ou Buret, et qui nourrit toute la littérature panoramique, quand la description bascule dans le décryptage et le dévoilement des "drames inconnus" (Soulié) qui courent sous la surface du social. C'est dans ces décryptages, ces dévoilements des envers et des mystères sociaux, dans cette injonction de l'élucidation, que s'esquisse une commune problématique du signe, de l'indice, une " sémiologie » et une «symptomatologie » du social ${ }^{12}$.

Il faut donc souligner l'inscription de nombreux romans de la Monarchie de Juillet dans une entreprise collective et multiforme d'écriture du social. La littérature panoramique, qui mélange sans souci les statistiques et les descriptions les plus sérieuses à la satire ou à la pochade, souligne, dans une mise à distance ironique, l'équivalence de tous les discours, et l'obsession du siècle à se représenter : elle propose ainsi une tentative de représentation, en même temps qu'une réflexion sur les moyens de la représentation et sur l'obsession représentative. Certains observateurs sociaux ne cessent de rechercher la bonne manière de rendre compte d'une misère partout insaisissable, de crimes obscurs, de plaies souterraines. Loin d'un Parent-Duchâtelet qui s'attache en premier lieu aux méthodes de l'investigation, insiste sur la nécessité des vérifications incessantes et préfère le chiffre à tous les témoignages ${ }^{13}$, d'autres observateurs sociaux s'inquiètent du mode de représentation adéquat à leur objet, quand la statistique s'avère impossible ou insuffisante : Frégier use et abuse de la typisation pour rendre sensible la jonction entre l'indigence et la tendance au crime ; Eugène Buret cherche à promouvoir une écriture du témoignage, mais semble tenté par l'écriture littéraire. Tout en opposant une écriture honnête, qui rendrait compte des «impressions [...] reçues directement de la vue des choses ", aux illusions de ces peintures étudiées, usant des « savants effets de l'art », il se demande si la plume des véritables écrivains ne serait pas plus adéquate pour décrire « la pittoresque vérité de l'extrême misère ${ }^{14}$. La sociologie descriptive flirte alors avec le 
vieux modèle de l'imitation, quand l'enquêteur propose de "copier, d'après nature, en citant nos témoins, quelques scènes empruntées au paupérisme des nations riches ${ }^{15}$.

En somme, ici et là, et comme ne cessent de le dire les préfaces balzaciennes, c'est toujours la même question qui est posée : comment représenter la complexité du social ? De nombreux romans de la Monarchie de Juillet partagent ainsi, avec l'enquête sociale et la littérature panoramique, un programme - rendre le monde social lisible - , des modalités descriptives - la typisation, l'articulation d'une figuration de la société comme surface à parcourir et profondeur à percer - qui renvoient, plus fondamentalement, à une interrogation sur le mode de représentation adéquat de la complexité du social.

Cette proximité des romans et des autres tentatives de représentation du social dans les années 1830-1840 dessine un terrain d'analyse commun à l'histoire des textes littéraires et à l'histoire des représentations. Isolant les textes littéraires des autres domaines du discours, l'analyse littéraire tend d'ordinaire à insister sur tout ce qui, dans le roman par exemple, se fait réécriture d'autres écritures : on peut ainsi envisager les descriptions balzaciennes comme un "mode d'affleurement» de cet "extra-texte» que forment d'autres systèmes de « mise en ordre » ou de « mise en classement », enquêtes sociales et médicales, classifications des sciences de la nature ou presse judiciaire ${ }^{16}$. L'ensemble de la littérature panoramique peut également être appréhendée comme une revue ironique de toutes les formes d'écriture du social, depuis le discours encyclopédique et le tableau statistique jusqu'au roman lui-même. Tout en marquant fortement le lien entre la description littéraire et les autres formes de mise en texte du monde, cette perspective constitue la littérature en un lieu radicalement autre, comme en surplomb. D'un autre côté, face au continent littéraire, les études historiques hésitent entre le pillage documentaire et la défiance : tantôt l'historien pioche dans les descriptions romanesques des «preuves » ou des illustrations de ses affirmations; tantôt il évite soigneusement toute incursion sur le terrain de la littérature, trop persuadé qu'une matière aussi subtile ne peut que lui glisser entre les doigts. Il faut pourtant poser la question de la représentation de la complexité de la société sous la monarchie de Juillet et souligner le rôle du roman, à côté de l'observation sociale et de la littérature panoramique, dans la production de visibilité et d'intelligibilité du social. Ces romans qui investissent la société contemporaine offrent des écritures, toutes spécifiques, du monde social: ils en présentent des figurations complexes - plus complexes peut-être, différemment complexes en tout cas -, que ce que propose l'enquête sociale à la même époque. Certes, ces figurations passent par des jeux de réécriture d'autres formes de décryptage du social. Sue joue sur cette ambiguïté lorsqu'il transforme le lieu de publication des Mystères de Paris, le feuilleton des Journal des Débats, en tribune de la réforme sociale, à partir de février 1843 : il déborde le cadre de la fiction, en proposant explicitement à ses lecteurs, dans les pauses extra-narratives du roman comme dans certaines plages descriptives, une parole en prise immédiate sur la réalité sociale. Ces phénomènes de frottement, de circulation, de concurrence entre domaines textuels, qui tiennent également à la mobilité des producteurs de tous ces textes entre littérature, journalisme, et enquête sociale, suggèrent, nous semble-t-il, un questionnement commun aux études littéraires et historiques ${ }^{17}$.

18 Les romans des années 1830-1840 ne constituent pas seulement des lectures du monde social par les projets qu'ils énoncent ou les procédés descriptifs qu'ils mettent en œuvre : s'ils s'inscrivent dans une entreprise collective et multiforme de réduction de l'opacité du monde social, il faut aussi souligner qu'ils sont reçus par leurs lecteurs comme des 
représentations pertinentes de la complexité sociale. Dans l'univers de lecture que suggère la réception de ces romans, chez les critiques de profession comme chez les lecteurs ordinaires, la vérité romanesque se situe bien du côté de la réalité sociale.

Si l'on définit par discours critiques l'ensemble des discours publiés (dans la presse ou en librairie) et tenus en public sur le roman sous la monarchie de Juillet, on se trouve confronté à une imposante masse discursive dont la richesse et le caractère hétéroclite suggèrent déjà l'intensité et la large extension des débats suscités par le roman : de fait, si tous les romans n'ont pas les honneurs de la presse, il n'est guère de journal qui ne consacre un espace à l'examen des romans nouveaux, ou qui n'intervienne dans le débat provoqué par les grands scandales romanesques; il n'est guère de revue littéraire ou de feuilleton critique qui ne donne de temps à autre un panorama de la librairie ou de la littérature contemporaine, largement consacré au roman, ou qui ne participe aux polémiques sur la littérature industrielle et le roman-feuilleton. La prise de parole sur le roman prolifère, en outre, hors de l'espace réservé des revues et des feuilletons littéraires, des livres pédagogiques ou religieux, ou du discours médical : dans toute la presse, féminine ou ouvrière par exemple, et au delà de la presse, dans les tribunaux ou à la Chambre, le roman devient l'objet d'un ample débat public.

Face à cette masse discursive, il faut, pour saisir ce qui se dit du roman, être attentif aux argumentations employées comme aux positions des individus qui interviennent publiquement. Comment parle-t-on du roman? L'emprise d'un idiome hérité des catégories et des schémas de jugement de la poétique classique constitue un premier élément commun à la plupart des prises de parole critique sur le roman : la tension entre vraisemblance et moralité, l'injonction du naturel, la question de la visée instructive ou édifiante dominent le discours critique. L'invocation de la moralité et du bon goût permet à toute une presse - catholique et légitimiste, ou littéraire - de dénoncer la représentation du peuple, des bas-fonds et des vices de l'époque dans les romans. S'énonce alors un ordre du représentable qui borne étroitement le territoire romanesque : les « plaies sociales » ne doivent pas être montrées sans précaution, et surtout pas dans le roman, dont la lecture, selon une opinion largement partagée, suscite avant tout l'imitation. Condamnant Un Grand homme de province à Paris, de Balzac, dans la Revue de Paris en juillet 1839, Jules Janin présente une argumentation caractéristique de ce type de position : «Mais il ne s'agit pas ici du talent de M. de Balzac. [...] Il ne s'agit pas de son esprit ; bien peu en ont autant que lui aujourd'hui, et personne n'en a davantage : il s'agit de ce que vous me disiez tout à l'heure, à savoir, s'il n'y avait pas, en effet, en dessous et en dehors du journal et dans une fange verdâtre où ils se cachent à tous les yeux, certains animaux venimeux qui jettent à tout venant leur bave immonde? Je vous dirai que je l'ignore, mais que cependant la chose est possible; tous les états de la société sont représentés dans les bagnes de Toulon et de Brest, aussi bien que sur le livre d'or de la Légion d'honneur. Est-ce à dire cependant que le roman et la comédie se puissent occuper de ces vils héros incessamment courbés sous le mépris public ou sous le bâton de l'argousin? Dans son Histoire de la prostitution publique, M. Parent-Duchatelet, ce savant gentilhomme qui, par charité, a vécu dans les immondices; ce rigide chrétien de PortRoyal qui toute sa vie a vécu dans les plus mauvais lieux, par vertu! raconte qu'un jour, afin de compléter son horrible science du vice parisien, on le fit entrer dans une vaste maison ou plutôt dans une horrible fosse d'aisance où, sur une montagne de chiffons ramassés dans toutes les boues du royaume, dormaient pêle-mêle avec des voleurs une centaine de filles de joie. M. Parent-Duchatelet l'a vu, il le raconte, il faut le croire. Et 
cependant, parce que la chose existe, est-ce donc à dire que le roman et la comédie, le crochet à la main, se puissent occuper de ce pandemonium grouillant sur ce tas d'immondices? Non, non, il y a des choses qu'on ne doit pas voir et qui sont à peine permises au philosophe, à peine permises au moraliste, à peine permises au chrétien ? Un écrivain n'est pas un chiffonnier, un livre ne se remplit pas comme une hotte. On cache dans les entrailles de la terre les égouts et les sentines; pourquoi donc voulez-vous les porter dans vos livres, pourquoi donc voudriez-vous faire de la littérature de ce pays un vaste cloaque, où chaque excrément du cœur, où chaque résidu de l'âme humaine serait apporté en triomphe?»

21 Le deuxième élément fondamental du discours critique sur le roman, sous la monarchie de Juillet, réside, nous l'avons suggéré, dans la tentative de constitution d'une sphère critique régulatrice de la production imprimée dans les revues littéraires ou dans les feuilletons des quotidiens. À mesure que la production romanesque se diffuse plus largement et atteint un nouveau public, surtout après la création du roman-feuilleton en 1836, s'invente et se fortifie un discours qui dénonce les tyrannies du tirage et la marchandisation de la littérature. On reprend alors les vieux préceptes de la vraisemblance et de la moralité pour mettre en cause cette littérature "industrielle", odieuse et sans style, et promouvoir, comme contre-modèle, les "romans intimes", comme ceux de Jules Sandeau, peignant les mœurs de gens de qualité pour un public de qualité, dans un modèle de circulation où la critique serait reine de la presse, et le roman objet de librairie.

La critique littéraire de la Monarchie de Juillet s'ordonne ainsi autour de deux motifs majeurs: l'interdiction de la représentation romanesque des plaies sociales et la dénonciation de la littérature industrielle. On peut percevoir, dans ce double anathème, toutes les anxiétés que suscitent tant les ambitions des romanciers à déchiffrer le contemporain que les usages, réels ou fantasmés, de leurs ouvrages par un public élargi à ses composantes les plus vulnérables : les femmes, la jeunesse, le peuple.

La troisième spécificité de l'époque tient au fait que les romans deviennent l'objet d'un discours proliférant, hors des lieux de la presse littéraire, religieuse ou pédagogique. Le scandale suscité par les Mystères de Paris en 1842-1844 permet, par exemple, d'en prendre la mesure. Les romans, par leur diffusion accrue et par leur volonté de représenter le contemporain, tendent à devenir un problème social et politique et non plus seulement une menace esthétique et morale. Au-delà même de l'affaire Lafarge ${ }^{18}$, la question des effets de la lecture des romans devient ainsi un thème fréquent du discours judiciaire : on relie le vol ou le meurtre aux représentations romanesques des inégalités de fortune, on rattache l'adultère et le crime passionnel à la dénonciation romanesque de la tyrannie du mariage ${ }^{19}$. À la Chambre des Députés, le baron de Chapuys-Montlaville prononce, entre 1843 et 1847, plusieurs discours contre le roman-feuilleton, à l'occasion des débats sur les lois sur la presse. Il déplore la vulgarité, la violence, le cynisme de ces textes : leur mise en cause des « institutions les plus saintes, [des] croyances les plus révérées » doublée de la représentation de richesses fabuleuses détourne l'homme de la "réalité des choses ». Il ne s'agit pas seulement d'une condamnation de l'invraisemblance des romans: le député de Saône-et-Loire s'inquiète de la manière dont les individus appréhendent leur position sociale à la lumière de la lecture des romans. En 1847, il souligne en effet que « la lecture habituelle de ces romans, en dégoûtant [...] chaque citoyen de sa situation et en lui faisant concevoir pour son avenir des espérances chimériques, cette lecture provoque le déclassement, en dérangeant le point où chacun doit placer sa dignité personnelle [...]. 
Pour ceux dont la tête est encombrée de chimères, leur dignité, l'honneur de leur vie, ne peuvent être satisfaits que par des situations éblouissantes. Il leur faut de la lumière, de l'éclat, de la puissance, des plaisirs, de l'or, et c'est pour cela qu'ils accumulent effort sur effort pour sortir de la condition de leur père, et s'élever vers les sommités; et comme chez la plupart les forces manquent à de tels desseins, ils retombent épuisés, ou bien ils se jettent dans les partis extrêmes et cherchent à ébranler les bases d'une société qui n'a pas été assez généreuse pour leur donner à chacun des portefeuilles et des millions. Cela conduit au déclassement, maladie épidémique de ce temps, je suis bien aise de le dire ici. Il est bien insensé celui qui cherche, par un sentiment de vanité maladroite, à échanger la veste ou le simple habit de village de ses pères pour les vêtements élégants des grandes villes; au lieu de s'élever, il s'abaisse, etc. ${ }^{20}$... Ce souci de maintien de l'immobilité de l'ordre social était déjà perceptible dans son discours de 1845 : «Si les romans précipitent la jeunesse riche dans la débauche et dans une farouche indépendance, oublieuse de tout devoir, ils exercent d'autres ravages sur la jeunesse des classes ouvrières: ils lui apprennent à mépriser la condition de leurs pères et à rougir de leur origine ; ils faussent ainsi la notion la plus simple et malheureusement la moins répandue de l'égalité ${ }^{21}$.

On ne saurait mieux dire la peur suscitée par l'appréhension des fonctionnements sociaux dans des romans soucieux, précisément, de représenter la complexité d'une société dont l'égalité constitue un principe problématique.

L'exploration des discours critiques sur le roman permet ainsi de saisir les modalités multiples par lesquelles se pense, souvent dans une tonalité dénonciatrice, le rapport aigu du roman au siècle. Elle indique également un éventail de lectures possibles des romans : les discours critiques proposent au public des modèles de lecture et de jugement légitimes; ils définissent ce en quoi le roman peut provoquer louange, condamnation, controverse ou scandale; quel type de pertinence, ou d'impertinence, peut lui être reconnu. Il faut ainsi prolonger cette étude de la réception critique des romans de la Monarchie de Juillet par celle des appropriations singulières des romans par leurs lecteurs, telles que le courrier adressé aux romanciers permet de les saisir.

Les remarques qui suivent s'appuient essentiellement de l'analyse de trois corpus de lettres de lecteurs : un ensemble de lettres adressées à Balzac entre 1830 et 1845, encore partiellement inédit ${ }^{22}$; le courrier des lecteurs adressé à Eugène Sue pendant la publication des Mystères de Paris ${ }^{23}$; un dossier de lettres inédites, adressées à Eugène Sue à la fin des années 1840, pendant la publication du Juif errant, de Martin l'enfant trouvé et des Sept péchés capitaux ${ }^{24}$.

Ces lettres apportent un éclairage essentiel, quoique partiel, sur la réception de certains des romans majeurs de la période : éclairage partiel, puisque même si les lecteurs qui écrivent aux romanciers sont issus des milieux sociaux les plus variés - de l'ouvrier à la duchesse, en passant par la bourgeoise de province -, ces individus donnent à la lecture une place si exceptionnelle dans leur existence qu'elle justifie l'engagement dans une relation épistolaire peu commune, qui traverse l'espace social en direction d'un inconnu glorifié ${ }^{25}$. En outre, ces lettres ne relatent pas seulement une intense expérience de lecture : elles formulent aussi, le plus souvent, des demandes d'argent, de conseils, de recommandations à ce personnage supposé puissant qu'est le romancier célèbre. Le discours sur la lecture qui s'y déploie est ainsi nécessairement pris dans des stratégies. $\mathrm{Si}$ on ne peut les tenir pour représentatives de la réception des romans de Balzac et de Sue dans le grand public, ces lettres suggèrent néanmoins certaines des modalités possibles de l'appropriation de ces romans. 
28 La lettre au romancier est d'abord le lieu d'une reconnaissance de la vérité de la représentation de la société que donnent les romans. Autour de personnages ou de situations romanesques, les lecteurs s'arrêtent sur la vérité de certaines configurations sociales données à lire par le roman : ce sont, dans le courrier de Balzac, les types de la vieille fille, de la femme de province incomprise ou du génie pauvre et ignoré ; dans le courrier de Sue, la misère méritante et ignorée d'artisans sur le modèle de Morel. La reconnaissance de la vérité des configurations sociales décrites dans le roman conduit à un décodage du monde du lecteur à la lumière du roman. Ce décodage de l'expérience sociale à la lumière du roman se déploie dans des récits de vie, dans une écriture de soi composée en référence au monde du roman, par une appropriation et un bricolage de schèmes romanesques.

29 En dépit de la dissemblance des univers romanesques de Sue et de Balzac, les lettres de leurs lecteurs fonctionnent ainsi de manière similaire : elles reposent sur une lecture très sérieuse des romans, comme représentations de la société contemporaine. Les lecteurs n'ignorent pas le caractère fictionnel des textes, mais s'attachent à la vérité sociale contenue dans la fiction; ils affirment que les romans offrent une représentation juste de la société contemporaine et déploient une écriture qui, dans une appropriation mimétique des éléments romanesques, leur permet d'énoncer identités et appartenances sociales complexes. À partir de la lecture des romans, les épistoliers formulent leurs propres itinéraires sociaux et disent souvent, dans ces récits de vie qui recomposent les éléments romanesques, une intense souffrance sociale, associant les malheurs individuels à des fonctionnements sociaux. Louise Abber, qui écrit à Balzac en 1836, formule ainsi, dans le même mouvement, sa douleur de vivre et la reconnaissance de la vérité romanesque: "j'ai énormément souffert, souvent vous avez fait vibrer en moi mille cordes douloureuses ", déclare-t-elle ${ }^{26}$. Elle adresse à Balzac une écriture à la troisième personne, qui peut se lire en miroir des textes balzaciens du début des années 1830 : elle a fait un malheureux mariage d'argent et dit avoir subi, pendant quinze ans, « les affreuses conséquences qu'une grande ville impose à la malheureuse femme d'un mari à passions trop vives et toutes physiques $"{ }^{27}$. Sa souffrance s'inscrit donc dans une configuration sociale spécifique, celle du mariage bourgeois, dénoncé par Balzac dès la Physiologie du mariage, en 1829. L'écriture de soi, chez cette lectrice, reprend ensuite des motifs balzaciens : « elle pleurait toujours, tout était devenu terne et froid, une maladie nerveuse et sanguine la saisit, l'ennui, le dégoût de la vie vinrent peser lourdement sur son cœur, et en arrêter les battements. On cria à l'anévrisme, pauvres gens ! / Un homme fut alors reçu chez elle. Sa position l'admit dans son intimité ; il se dit malheureux, elle le plaignit, il se dit souffrant, elle souffrit de ses souffrances, il l'aima, elle aussi l'aima! » ${ }^{28}$

Comme les héroïnes du Rendez-vous (La Femme de trente ans) ou du Lys dans la vallée, Louise est prise de « cet incroyable besoin d'aimer » ${ }^{29}$ au moment où « l'amour permis, l'amour conjugal s'évanouissait au milieu de graves souffrances physiques et morales $"{ }^{30}$; comme Julie d'Aiglemont accueille Charles de Vandenesse "dans le sanctuaire du ménage ", Louise reçoit un homme dans son intimité. Et, comme chez Balzac, l'amour naît dans la confidence du malheur: un amour irrésistible car, comme le souligne le narrateur balzacien, «il est impossible à une femme, à une épouse, à une mère, de se préserver contre l'amour d'un jeune homme " ${ }^{31}$. Louise Abber parle, avec Balzac, de la solitude et des souffrances d'une bourgeoise mariée selon les lois de l'intérêt, et non du cœur. La «femme de trente ans » constitue ainsi un type social et moral à partir duquel la lectrice peut décrypter et formuler sa vie. 
31 Avec l'aide des romans, bien des lecteurs mettent en récit des itinéraires marqués par des décalages: chutes, ruines, abandons ou échecs. Au delà des différences des milieux d'origine et des situations actuelles des scripteurs, la distance entre les aspirations ou les origines et la position sociale présente est au principe de nombreuses lettres : se pressent ainsi auprès des romanciers des étudiants ou des jeunes gens en quête de célébrité littéraire ou des écrivains manqués qui ne cherchent plus qu'à assurer leur sécurité matérielle. Ici s'énonce une souffrance de l'obscurité, de la méconnaissance. Julien Lépinay, jeune pion et apprenti poète qui écrit à Balzac pour obtenir quelques francs, souligne ainsi que sa misère matérielle n'est rien à côté de cette misère morale que provoque son obscurité : «Oh Monsieur de Balzac, votre jeunesse n'a pas été heureuse à ce que je vois, mais n'accusez pas le sort, si vous saviez... et qu'était-ce que votre malheur? Au moins vous vous êtes trouvé dans des circonstances où votre imagination pouvait travailler, se développer, créer, enfanter. Votre malheur, votre pauvreté avaient quelque chose de grandiose, d'aventureux, d'héroïque, mais moi... de ces petites misères, si petites, si vulgaires, si épicières... / Vous, on pouvait vous plaindre, parce que vous intéressiez, mais moi qu'ai-je d'intéressant dans ma petite infortune, si petite, si misérable, si pauvre petit menu peuple, si obscure ${ }^{32}$.

Ce sont, ailleurs, des individus aux itinéraires brisés par la faillite; des femmes seules ou abandonnées, des filles aux pères malades, des mères sans appui. Souffrances bourgeoises ou petites-bourgeoises qui ne sont pas tant des souffrances de la pauvreté elle-même, que du mouvement qui y mène, et du silence, de l'ignorance qui entoure la misère. C'est la force du roman que de rendre lisible et dicible l'inscription des destinées individuelles dans un espace social brouillé. Les bouleversements issus de la Révolution, la tension entre le principe de l'égalité et le fait de l'inégalité, ont produit de l'opacité sociale et de la souffrance ; le roman permet de formuler l'ambition, l'échec, la ruine, de nommer les formes de la mobilité des individus dans l'espace social. L'écriture aux romanciers dit ces souffrances obscures qui prennent naissance dans les fonctionnements opaques de cette société que représentent les romans de Sue et de Balzac. Cette écriture permet également de soulager, très concrètement, ces souffrances du décalage et de la mobilité sociale descendante: de nombreux lecteurs utilisent en effet la lettre à l'écrivain pour tâcher de sortir de l'obscurité de leur condition. Il s'agit, pour les uns, de parvenir, avec l'aide des romanciers, à la célébrité littéraire. D'autres, à l'image de Mme Hanska, tentent d'entrer dans la sphère magique de l'entourage du grand écrivain, en devenant l'un de ses correspondants privilégiés. Certains attendent enfin que les romanciers publient leurs récits de vie en les intégrant à leurs œuvres ou en les prenant pour base d'œuvres futures : leurs itinéraires prendront alors l'épaisseur des destinées romanesques. Tous ces lecteurs essayent de sortir de ces conditions sociales vécues comme brouillées, en entrant, sur des modes multiples, en rapport avec le monde de la littérature. Les lettres aux romanciers attestent ainsi sans cesse de cette capacité reconnue au roman de décrire et de décrypter le monde social dans sa complexité, mais signalent également le pouvoir attribué à la littérature de briser l'obscurité des conditions sociales, non seulement en les rendant lisibles, mais en formant le lieu social où cette obscurité semble pouvoir s'effacer. Comme lieu de représentation du social, le roman suscite une forme de désir de représentation - désir d'être représenté, désir de participer à la représentation, d'accéder à la parole sur le monde contemporain. Ce désir ne renvoie pas seulement au statut social envié des hommes de lettres mais à la perception du brouillage du monde, ce monde privé de la lisibilité que lui donnerait la représentation démocratique. Comme si, 
en l'absence de représentation politique directe, le roman pouvait constituer le lieu textuel d'une représentation de la société contemporaine. romans sur la société contemporaine sous la monarchie de Juillet, nous avons voulu sortir du paradigme qui guide fréquemment l'analyse du contenu des textes littéraires en histoire et en histoire littéraire : paradigme indiciaire au juste, qui hérite de la conception romantique de la littérature comme expression d'une âme, des mœurs d'un milieu ou d'une époque tout entière. De ce paradigme relève l'usage de la littérature comme document sur la réalité sociale: la littérature porterait la trace du passé, et la critique historienne du texte littéraire devrait permettre de remonter vers cette réalité. Relève également de cette conception l'usage que l'histoire des mentalités faisait des textes, alors envisagés comme traces de la vision du monde d'un groupe social. L'histoire des représentations s'appuie également sur ce paradigme, quoique de manière plus complexe, lorsqu'elle traque dans les textes littéraires des représentations qui sont ellesmêmes des traces d'autres représentations plus diffuses, que la littérature contribue en retour à façonner. Les représentations romanesques, par exemple, constituent ainsi des lieux d'agrégation de représentations, et certaines situations ou certains personnages romanesques permettant de pénétrer dans la « conflictualité des représentations » ${ }^{33}$. réalité sociale passée, des expressions de l'esprit d'une époque, ou des lieux d'agrégation de représentations diffuses et parfois contradictoires, nous avons essayé de comprendre comment les romans sur la société contemporaine de la monarchie de Juillet ont pu constituer, dans le temps de leur production et leurs usages, comme un lieu, une " configuration spécifique de la vérité » ${ }^{34}$.

Dans un article exemplaire des usages documentaires du roman balzacien, Louis Chevalier, après avoir longuement ausculté la vérité historique de la Comédie humaine à la lumière des acquis de l'histoire quantitative, s'éloignait de la question de «l'authenticité » de la description balzacienne pour se demander si la plus grande force de la Comédie humaine ne résidait pas dans sa vérité architecturale: la vérité de sa " conception", de son "invention", de sa "structure", de sa "lumière et de ses mystères " ${ }^{35}$. Le problème de l'historien, soulignait-il alors, doit être de "comprendre comment, à partir de cette observation consciente et inconsciente, dévorante et passive » de son temps, Balzac a « décrit et recréé, jusqu'à donner l'impression d'imiter la nature, d'apporter non un roman, mais un document ${ }^{36}$. Le système balzacien " correspond 
parfaitement aux exigences de l'histoire et de la description sociale ", la Comédie humaine "apporte à l'histoire et à la société leur programme et leur modèle " ${ }^{37}$ : Chevalier se détourne donc de la question de la valeur documentaire de l'opus balzacien pour s'intéresser à la proximité troublante de l'écriture romanesque balzacienne, dans sa construction et ses procédés, avec ce que devrait être celle de l'histoire sociale. Il insiste tout particulièrement sur la vision constructiviste des groupes sociaux chez Balzac: «Balzac nous apprend qu'il n'est pas de définition en soi des classes sociales, ni, pour leur étude, de méthode applicable indifféremment à toutes les classes et en tous temps $"{ }^{38}$. Balzac apporte donc le modèle d'une démarche de sociologie compréhensive, comme il offre celui d'une écriture de la complexité du social, de l'articulation du singulier et du collectif, du biologique et du social. On ne saurait mieux suggérer l'inscription des romans de la Monarchie de Juillet dans l'histoire longue des savoirs et des écritures du social.

\section{NOTES}

1.. Ernest LEGOUVÉ, Soixante ans de souvenirs, Paris, Éditions J. Hetzel, 1886, tome 1, p. 370.

2.. Nora ATKINSON, Eugène Sue et le roman-feuilleton, Nemours, Éditions André Lesot, 1929, p. 100.

3.. Louis VILLERMÉ, Tableau de l'état physique et moral des ouvriers, Paris, J. Renouard, 1840, 2 volumes ; Charles FRÉGIER, Des classes dangereuses de la population des grandes villes et des moyens de les rendre meilleures, Bruxelles, Méline, Cans et Cie, 1840, 2 volumes ; Eugène BURET, De la misère des classes laborieuses en Angleterre et en France : de la nature de la misère, de son existence, de ses effets, de ses causes, et de l'insuffisance des remèdes qu'on lui a opposés jusqu'ici, avec les moyens propres à en affranchir les sociétés, Paris, Paulin, 1840, 2 volumes.

4.. Edmond WERDET, De la librairie française, Paris, Dentu, 1860.

5.. Martyn LYONS, Le triomphe du livre. Une histoire sociologique de la lecture au XIX siècle, Paris, Éditions Promodis/Éditions du Cercle de la Librairie, 1987, 302 p.

6.. La querelle du roman-feuilleton. Littérature, presse et politique, un débat précurseur (1836-1848), textes réunis et présentés par Lise DUMASY, Grenoble, Éditions littéraires et linguistiques de l'université de Grenoble, 1999, $280 \mathrm{p}$.

7.. L'expression, souvent reprise au XIX ${ }^{e}$ siècle, est empruntée à La Harpe, à propos du Gil Blas de Lesage ; LA HARPE, Lycée ou cours de littérature ancienne et moderne, Paris, Agasse, 1799 , tome 14.

8.. Sur la littérature panoramique, voir surtout Richard SIEBURTH, « Une idéologie du lisible : le phénomène des "Physiologies" ", dans Romantisme, n 47, 1985, pp. 39-60 ; et " Les Français peints par eux-mêmes ». Panorama social du XIXe siècle, catalogue rédigé et établi par Ségolène LE MEN et Luce ABÉLÈS, Paris, Éditions de la Réunion des musées nationaux, 1993, $109 \mathrm{p}$.

9.. Walter BENJAMIN, Das Passagen-Werk, Francfort-sur-le-Main, Éditions Suhrkamp, 1983, traduction française Paris, capitale du XIX ${ }^{e}$ siècle. Le Livre des Passages, Paris, Éditions du Cerf, 1989, $974 \mathrm{p}$. 
10.. Giovanna PROCACCI, Gouverner la misère, Paris, Éditions du Seuil, 1993, 357 p.

11.. Philippe HAMON, Du descriptif, Paris, Éditions Hachette, 1993, 247 p.

12.. Françoise GAILLARD, « Désordre social et ordre romanesque : une lecture de la théorie de la démarche ", dans Nineteenth Century French Studies, 1993, volume 21, n 3-4, pp. 277-291. En dépit de leur importance, il faut souligner que les configurations descriptives et narratives de l'enquête et de l'élucidation ne sont qu'un des modes de déchiffrement mis en œuvre dans le roman, la littérature panoramique et l'enquête sociale. L'appréhension - et l'écriture - du social comme épaisseur, secret, mystère, ou obscurité ne constitue que l'un des aspects d'une entreprise qui cherche avant tout à figurer la complexité du social. Cette multiplicité des modes d'approches du social se perçoit dans les romans " à mystères `", comme les Mémoires du Diable ou les Mystères de Paris, qui représentent la société autant comme un labyrinthe tentaculaire à parcourir que comme un énigme, comme dans l'observation sociale : si Parent-Duchâtelet ou Villermé entendent bien appréhender le contemporain par l'enquête ; Frégier ou Buret penchent vers d'autres solutions, celle d'une représentation typisée du social pour le premier, ou la composition de témoignages pour le second. La mise en texte du monde social par le roman croise ainsi, sous la monarchie de Juillet, la diffusion du modèle de l'enquête, qui s'impose, à partir des années 1830, comme « le principal mode d'approche, mais aussi de compréhension et de production du savoir propre à la société moderne » ; voir Dominique KALIFA, « Usages du faux. Faits divers et romans criminels au XIX ${ }^{\mathrm{e}}$ siècle », dans Annales, Histoire, sciences sociales, novembre-décembre 1999, nº 6, pp. 1358-1360.

13.. Alexandre PARENT-DUCHÂTELET, La prostitution à Paris au XIX $X^{e}$ siècle, texte présenté et annoté par Alain Corbin, Paris, Éditions du Seuil, 1981 (1 ère édition 1836), 216 p.

14.. Eugène BURET, De la misère..., ouv. cité, tome 1, p. 366.

15.. Idem, p. 367.

16.. Philippe HAMON, Du descriptif..., ouv. cité., pp. 56-60.

17. Pierre Rosanvallon esquisse une perspective de ce type lorsque, s'intéressant à la mise en place, au XIX ${ }^{\mathrm{e}}$ siècle, d'une « économie générale de la représentation ", il souligne que « bien avant que ne soit formulé le projet plus scientifique d'une physique sociale, c'est à travers la littérature et l'essai que se cherchent des principes d'intelligibilité »; Pierre ROSANVALLON, Le peuple introuvable. Histoire de la représentation démocratique en France, Paris, Éditions Gallimard, 1998, p. 288.

18. En août 1839, Marie Cappelle, orpheline issue d'une famille de la bourgeoisie parisienne, avait été contrainte d'épouser un maître de forges supposé riche et inconnu d'elle, Charles Lafarge, et de s'installer près de la forge, dans sa belle-famille, en Limousin... Cinq mois après le mariage, Charles Lafarge était mort ; la jeune femme fut accusée de l'avoir empoisonné à l'arsenic. Le procès eut un grand retentissement, à cause de la bataille d'experts qui se révélèrent incapables de prouver l'empoisonnement, ainsi que du profil social et culturel de l'accusée : le cas de Mme Lafarge semblait exemplaire des aspirations démesurées que peuvent éveiller chez les filles de la bourgeoisie une éducation trop poussée et le goût de la littérature. Sur la mise en cause du roman à l'occasion de l'affaire Lafarge, voir les articles d'Alfred NETTEMENT dans La Gazette de France en 1840-1841, repris dans ses Études critiques sur le feuilleton-roman, Paris, Perrodil, 1845-1846, 2 volumes, ainsi que l'étude de Jann MATLOCK, « Lire dangereusement. Les Mémoires du Diable et ceux de Mme Lafarge », dans Romantisme, $\mathrm{n}^{\circ}$ 76, 1992, pp. 3-21. 
19.. Le caractère criminogène des romans devient un poncif dans la seconde moitié du siècle ; voir Dominique KALIFA, L'encre et le sang. Récits de crime et société à la Belle Époque, Paris, Librairie Arthème Fayard, 1995, 351 p.

20.. CHAPUYS-MONTLAVILLE, Discours à la Chambre des Députés, le 6 avril 1847, dans Le Moniteur Universel, 7 avril 1847 ; repris dans La querelle du roman-feuilleton..., ouv. cité, pp. 104-116.

21. CHAPUYS-MONTLAVILLE, Discours à la Chambre des Députés du 14 mars 1845, dans Le Moniteur Universel, 15 mars 1845 et La querelle du roman-feuilleton..., ouv. cité, pp. 95-103. 22. Voir Honoré de BALZAC, Correspondance, éditée par Roger Pierrot, Éditions Garnier, 1960-1969, 4 volumes et Marcel BOUTERON, « Lettres de femmes adressées à Balzac », dans Cahiers balzaciens, $\mathrm{n}^{\circ} 3$ et $\mathrm{n}^{\circ}$ 5, 1924 et 1927.

23.. Ce

dossier, conservé à la Bibliothèque historique de la Ville de Paris, a été publié Jean-Pierre GALVAN, Les mystères de Paris. Eugène Sue et ses lecteurs, Paris, Éditions L'Harmattan, 1998, 430 p. et 431 p.

24.. Papiers d'Eugène Sue, Bibliothèque mMunicipale d'Orléans.

25.. Les manuels épistolaires de l'époque ne comportent aucun modèle de lettre de lecteurs. Sur le corpus des manuels épistolaires, voir Cécile DAUPHIN, « Les manuels épistolaires au XIX ${ }^{\mathrm{e}}$ siècle », dans Roger CHARTIER [dir.], La correspondance, Paris, Librairie Arthème Fayard, 1991, pp. 209-272. En revanche, la plus célèbre des correspondances d'un écrivain avec l'une de ses lectrices, Gœthe et Bettina von Arnim, a été traduite en français. Balzac l'avait lue avant de rédiger, d'ailleurs sur une idée de Mme Hanska, le roman de Modeste Mignon; Gœthe et Bettina. Correspondance inédite de Gœthe et de Mme Bettina d'Arnim, traduit de l'allemand par Sébastien Albin, Paris, Au comptoir des imprimeurs unis, 1843.

26.. Lettre de Louise Abber à Balzac, Fonds Lovenjoul, Bibliothèque de l'Institut, publiée par Marcel BOUTERON, «Lettres de femmes adressées à Balzac », dans Cahiers balzaciens, $\mathrm{n}$ -3, 1924, p. 31. Louise Abber est un pseudonyme.

27.. Idem, p. 43.

28. Idem, p. 44.

29.. Idem, p. 43.

30.. Honoré de BALZAC, La femme de trente ans, Paris, Le Livre de Poche, 1991, p. 74. Le passage cité provient du Rendez-vous, nouvelle d'abord publiée dans la Revue des Deux Mondes de septembre-octobre 1831, avant d'être réunie à d'autres textes, dans les Scènes de la vie privée de 1834, pour former un ensemble intitulé Même histoire qui, remanié, devint le roman de La femme de trente ans dans la Comédie humaine.

31.. Idem, p. 140 ; il s'agit du chapitre 3, « À trente ans », qui reprend la nouvelle intitulée La Femme de trente ans publiée dans la Revue de Paris en avril 1832.

32. Julien Lépinay à Balzac, Paris, le 23 novembre 1836. Lettre inédite, Fonds Lovenjoul, Bibliothèque de l'Institut.

33. Michèle RIOT-SARCEY, « De la représentation », dans Romantisme, $\mathrm{n}^{\circ} 110,2000$, p. 4. 34. Christian JOUHAUD, « Histoire et histoire littéraire », dans Henri BÉHAR et Roger FAYOLLE [dir.], L'Histoire littéraire aujourd'hui, Paris, Librairie Armand Colin, 1990, pp. 162-175.

35.. Louis CHEVALIER, « La Comédie humaine, document d'histoire », dans Revue historique, juillet 1964, pp. 27-48.

36. Idem, p. 45.

37.. Idem, p. 43. 
38.. Idem, p. 44.

\section{RÉSUMÉS}

Cet article tente d'apprécier le rôle du roman comme entreprise de description et de décryptage sous la Monarchie de Juillet. La production romanesque partage alors avec les autres écritures du social - enquêtes sociales, tableaux de Paris ou "physiologies" sociales - les mêmes questions; elle recourt aux même procédures descriptives et "décryptives". Critiques littéraires et simples lecteurs reconnaissent, sur des modes variés, la vérité sociale de ces représentations romanesques du contemporain. Replacés dans l'économie discursive de leur époque, et envisagés dans les appropriations qu'ils suscitent, les romans de la Monarchie de Juillet peuvent ainsi être appréhendés dans la perspective d'une histoire longue des savoirs et des écritures du social.

The novelist as a social observer in France under the July Monarchy. This article assesses novels published during the July Monarchy as attempts to describe and decipher society. Novels shared then many common features with other types of writings about society, such as social surveys, "tableaux de Paris" or social "physiologies": they dealt with the same questions and resorted to the same descriptive and "decryptive" procedures. Literary critics as well as ordinary readers acknowledged in various ways the social veracity of literary representations of the contemporary world. Once the novels of the July Monarchy are considered within the general discourse of their time and through readers' appropriations, their study can be a building block of a broader history of the forms of social knowledge.

\section{AUTEUR}

\section{JUDITH LYON-CAEN}

Assistante-monitrice normalienne à l'Université Paris 10-Nanterre 